\title{
A RIGHT PCI RING IS RIGHT NOETHERIAN
}

\author{
ROBERT F. DAMIANO
}

\begin{abstract}
C. Faith and J. Cozzens have shown that a ring, whose right proper cyclic modules are injective, is either semisimple or a simple, right semihereditary, right Ore $V$-domain. They have posed a question as to whether such a ring is right noetherian. In this paper, an affirmative answer is given to that question. Moreover, necessary and sufficient conditions are given as to when a right PCI ring is left PCI.
\end{abstract}

In [1], Faith and Cozzens proved that a ring $R$ whose proper right cyclic modules are injective must be either semisimple or a simple, right semihereditary, right Ore $V$-domain. They noted that all the known examples of such rings are right noetherian and posed the question whether every ring with this property is noetherian. We will answer this question in the affirmative. More clearly, we will show:

THEOREM 1. Let $R$ be a right PCI ring, then either

(a) $R$ is semisimple, or

(b) $R$ is a simple right noetherian, right hereditary, right Ore $V$-domain.

By using Boyle's theorem [1, Theorem 6.26] and Theorem 1, we get the following immediately:

COROllary. $R$ is a PCI ring, that is a right and left PCI ring, if and only if $R$ is either semisimple or a simple noetherian hereditary Ore $V$-domain.

Proof of Theorem. Faith and Cozzens have shown that a PCI ring $R$ must be either semisimple or a simple, right semihereditary, right Ore $V$-domain [1, Theorems $6.13,6.17]$. Thus, we may assume $R$ is a simple, right semihereditary, right Ore $V$-domain that is not semisimple. Furthermore it is known that such a ring has the property that every proper finitely presented cyclic module has a von Neumann regular endomorphism ring [1, Proposition 6.20].

Suppose that the endomorphism ring of every proper finitely presented cyclic module is semisimple. Then, every proper finitely presented cyclic module has an indecomposable decomposition. It is clear from the properties of $R$ that the only indecomposable injective modules are the simple modules and $E\left(R_{R}\right)$. Thus, it follows that every proper finitely presented cyclic module is a direct sum of a semisimple module and a finite number of isomorphic copies of $E\left(R_{R}\right)$.

Received by the editors October 5, 1978 and, in revised form, January 22, 1979.

AMS (MOS) subject classifications (1970). Primary 16A48. 
If there exists a proper finitely presented cyclic module that is not semisimple, then $E\left(R_{R}\right)$ is finitely presented cyclic. However, it is known that this implies $R$ is semisimple [1, Theorem 6.17]. Thus, every proper finitely presented cyclic module is semisimple.

In particular, this implies that every proper right ideal of $R$ is finitely generated. Hence, $R$ is right noetherian and we are done.

Thus, it will suffice to prove the following.

Proposition. Let $R$ be a ring whose proper right cyclic modules are injective. Let $x R$ be a proper finitely presented cyclic module and $S=\operatorname{End}(x R)$. Then $S$ is a semisimple ring.

Proof. We have that $S$ is a von Neumann regular ring. It is clear that it suffices to prove that $S$ does not contain an infinite set of orthogonal idempotents.

Thus, suppose that $\left\{e_{i} \mid i \in \mathcal{G}\right\}$ is a set of nonzero orthogonal idempotents in $S$. Let $A \subseteq \mathcal{G}$. We note that since $x R$ is injective, $E\left(\sum_{i \in A} e_{i} x R\right)$ is a direct summand of $x R$. Thus, there exists a nonzero idempotent $E_{A} \in S$ such that

$$
E_{A} x R=E\left(\sum_{i \in A} e_{i} x R\right)
$$

Clearly,

$$
E_{A} e_{i}=e_{i} \quad \forall i \in A \text {. }
$$

Let $j \in g-A$. Suppose $e_{j} E_{A} x R$ is a nonzero cyclic submodule of $x R$. Since $S$ is von Neumann regular, there exists a nonzero idempotent $e \in S$ such that

$$
S e_{j} E_{A}=S e .
$$

Since $\sum_{i \in A} e_{i} x R \triangleq E_{A} x R$, there exists $0 \neq r \in R$ such that

$$
0 \neq y=E_{A} \text { exr } \in \sum_{i \in A} e_{i} x R .
$$

Therefore, $0=e_{j} y=e_{j} E_{A}$ exr. Since $e_{j} E_{A} \in S e$, this implies that $e_{j} E_{A} x r=0$. Similarly since $e \in S e_{j} E_{A}$, this implies that exr $=0$ and hence $y=0$. Thus, $E_{A}$ ex $R=0$. In particular, we get $e_{j} E_{A} x R=e_{j} E_{A}$ ex $R=0$. Hence,

$$
e_{j} E_{A}=0 \quad \forall j \in \mathscr{G}-A \text {. }
$$

We now use a modification of a constructive technique that appears in the proof of Osofsky's thoerem [3]. Let

$$
\mathscr{g}=\bigcup_{A \in \mathscr{Q}} A
$$

where $\mathscr{U} \subseteq 2^{\mathscr{g}}$, the power set of $\mathcal{F}$, is infinite such that for all $A, B \in \mathcal{U}, A$ is infinite and $A \cap B \neq \varnothing$ if and only if $A=B$. By Zorn's lemma, the $\mathcal{Q}$ can be enlarged to a set $\mathscr{B} \subseteq 2^{\mathscr{g}}$, the power set of $\mathcal{G}$, with respect to the properties: 
(i) if $A \in \mathscr{B}, A$ is infinite,

(ii) if $A, B \in \mathscr{B}, A \neq B \Rightarrow A \cap B$ is finite.

Let

$$
N=\left\{u \in x R \mid e_{i} u=0 \text { for all but finitely many } i \in \mathcal{G}\right\} .
$$

We claim that $x R / N$ is not injective, which will give us a contradiction and complete the proof. Let $A \in \mathscr{B}, x r \in x R$. Assume that $E_{A} x r \notin N$, where $E_{A}$ is as previously defined. However by the construction of $\mathscr{B}$, if $A_{j} \subseteq \mathscr{B}_{-}$ $\{A\}, 1 \leqslant j \leqslant n$, then $A \cap\left(\cup_{i=1}^{n} A_{j}\right)$ is finite. Thus, for all but a finite number of $i \in A, e_{i} E_{A_{j}}=0,1 \leqslant j \leqslant n$. Therefore $E_{A} x r \notin \sum_{j=1}^{n} E_{A_{j}} x R+N$. Thus $\Sigma_{A \in \mathscr{B}}\left(E_{A} x R+N\right)$ is direct in $x R / N$.

Define the homomorphism

$$
\varphi:\left(\sum_{A \in \mathscr{B}} E_{A} x R+N\right) / N \rightarrow x R / N
$$

by

$$
\varphi\left(E_{A} \bar{x}\right)=E_{A} \bar{x}, \quad A \in \mathcal{Q},
$$

and

$$
\varphi\left(E_{A} \bar{x}\right)=0, \quad A \in \mathscr{B}-\mathscr{Q},
$$

where $\bar{x}$ is the image of $x$ in $x R / N$. Assume that $x R / N$ is injective. Then $\varphi$ extends to a homomorphism $\bar{\varphi}: x R / N \rightarrow x R / N$.

Let $\bar{\varphi}(\bar{x})=\bar{x} r$. Since $x R$ is cyclic, we can choose an $r_{A} \in R$ such that $E_{A} x=x r_{A}$. Then, since $\bar{\varphi}$ extends $\varphi, \bar{\varphi}\left(\bar{x} r_{A}\right)=\bar{x} r r_{A}=\bar{x} r_{A}$.

Thus $x r r_{A}=x r_{A}+u$ where $u \in N$. Again since $x R$ is cyclic, we let $r_{i} \in R$ such that $e_{i} x=x r_{i}$. We note, with this notation, that since $E_{A} e_{i}=e_{i}$ for all $i \in A$, that $x r_{A} r_{i}=x r_{i}$, and that since $e_{i} e_{i}=e_{i}$ for all $i \in A, x r_{i} r_{i}=x r_{i}$. Thus

$$
x r r_{A} r_{i}=x r_{A} r_{i}+u r_{i}=x r_{i}+u r_{i}
$$

Since $u \in N$, there exists only a finite subset $\left\{i_{l} \mid i_{l} \in A, 1<l<n\right\}$ such that $e_{i} u \neq 0$. Let $i \in A-\left\{i_{l} \mid 1 \leqslant l \leqslant n\right\}$. Then

$$
e_{i} x r r_{A} r_{i}=e_{i} x r_{i}
$$

that is,

$$
x r_{i} r r_{A} r_{i}=x r_{i} r_{i}=x r_{i} .
$$

4 Let $A^{\prime}=\left\{i \in A \mid x r_{i} r r_{A} r_{i}=x r_{i}\right\} \neq \varnothing$. We note that by construction $A^{\prime}$ is an infinite set. Since $x R$ is finitely presented, $I_{R}=\operatorname{Ann}_{r}(x)=\{t \in R \mid x t=0\}$ is finitely generated, that is

$$
I=t_{1} R+\cdots+t_{k} R, \quad t_{j} \in R .
$$

Since $\bar{\varphi}$ defines an $R$-homomorphism, there exists only a finite number of $i \in \mathcal{g}$ such that $e_{i} x r t_{j}=x r_{i} r t_{j} \neq 0$ for each $t_{j}, 1 \leqslant j \leqslant k$. Let

$$
A^{\prime \prime}=\left\{i \in A \mid x r_{i} r r_{A} r_{i}=x r_{i} \text { and } x r_{i} r t_{j}=0,1 \leqslant j \leqslant k\right\} .
$$

It is clear by construction, that $A^{\prime \prime}$ is an infinite set. Let $C$ be a choice set from $\left\{A^{\prime \prime} \mid A \in \mathscr{U}\right\}$. By the maximality of $\mathscr{B}$, there exists $D \in \mathscr{B}-\mathscr{U}$ such 
that $C \cap D$ is infinite. But since $x r r_{D} \in N$ by the construction of $\bar{\varphi}$, there exists only a finite number of $i \in \mathcal{G}$ such that $e_{i} x r_{D} \neq 0$. Thus we can choose $i \in C \cap D$ such that

$$
e_{i} x r r_{D}=0
$$

Thus

$$
x r_{i} r r_{D} r_{i}=0 .
$$

However $x\left(r_{D} r_{i}-r_{A} r_{i}\right)=0$ implies that $r_{D} r_{i}-r_{A} r_{i} \in I$. Let $r_{D} r_{i}=r_{A} r_{i}+t$ where $t \in I$. Thus, since $i \in A^{\prime \prime}$,

$$
0=x r_{i} r r_{D} r_{i}=x r_{i} r r_{A} r_{i}+x r_{i} r t=x r_{i}+x r_{i} r t=x r_{i} \text {. }
$$

Thus $e_{i}=0$, a contradiction.

We note that it is still an open question whether a right PCI ring must be a left PCI ring. Faith and Cozzens [1, Theorem 6.25] have reduced the question to whether a right PCI ring is left Ore. It seems that a counterexample along the lines of $[1,7.10]$ has some possibility. However, we can supply a necessary and sufficient condition for a one-sided PCI ring to be two-sided PCI. In particular, we have:

Theorem 2. A right PCI ring $R$ is left PCI if and only if $R$ is left coherent.

Proof. By Theorem 1, one direction is clear. For the other, suppose $R$ is a left coherent, right PCI ring. Then, since $R$ is right nonsingular, $E\left(R_{R}\right)$ is flat [4, Corollary XI, 3.2]. Moreover, $R$ is of finite right rank and, hence, every finitely generated nonsingular right $R$-module can be embedded in a free module [4, Corollary XII, 7.3]. Since every finitely generated right torsion-free $R$-module is right nonsingular, it follows by Levy's theorem [2, Theorem 5.3] that $R$ is right Ore. By [1, Theorem 6.25], Theorem 1, and [1, Theorem 6.26], $R$ is left PCI.

ACKNOWLEDGEMENTS. I would like to thank the referee for several helpful suggestions, especially in regard to the organization of this paper. I would also like to thank Gary Roth for several stimulating discussions.

\section{REFERENCES}

1. J. Cozzens and C. Faith, Simple noetherian rings, Cambridge Tracts in Math. and Math. Phys., University Press, Cambridge, 1975.

2. L. Levy. Torsion-free and divisible modules over nonintegral domains, Canad. J. Math., 15 (1963), 132-157.

3. B. Osofsky, Noninjective cyclic modules, Proc. Amer. Math. Soc. 19 (1968), 1383-1384.

4. B. Stenström, Rings of quotients, Springer-Verlag, Berlin and New York, 1975.

Department of Mathematics, University of Oklahoma, Norman, Oklahoma 73069 